\title{
Stability of bunched fluxons in magnetically coupled Josephson junctions
}

\section{Grønbech-Jensen, Niels; Cai, David; Samuelsen, Mogens Rugholm}

\section{Published in:}

Physical Review B

Link to article, DOI:

10.1103/PhysRevB.48.16160

Publication date:

1993

Document Version

Publisher's PDF, also known as Version of record

Link back to DTU Orbit

Citation (APA):

Grønbech-Jensen, N., Cai, D., \& Samuelsen, M. R. (1993). Stability of bunched fluxons in magnetically coupled Josephson junctions. Physical Review B, 48(21), 16160-16163. https://doi.org/10.1103/PhysRevB.48.16160

\section{General rights}

Copyright and moral rights for the publications made accessible in the public portal are retained by the authors and/or other copyright owners and it is a condition of accessing publications that users recognise and abide by the legal requirements associated with these rights.

- Users may download and print one copy of any publication from the public portal for the purpose of private study or research.

- You may not further distribute the material or use it for any profit-making activity or commercial gain

- You may freely distribute the URL identifying the publication in the public portal 


\title{
Stability of bunched fluxons in magnetically coupled Josephson junctions
}

\author{
Niels Grønbech-Jensen and David Cai \\ Theoretical Division, Los Alamos National Laboratory, Los Alamos, New Mexico 87545 \\ Mogens R. Samuelsen \\ Physics Department, The Technical University of Denmark, 2800 Lyngby, Denmark
}

\begin{abstract}
The stability of bunched fluxon states in inductively coupled Josephson junctions is analyzed for fluxons of both equal and opposite polarity. We demonstrate that unipolar fluxons may form stable bunched states if the velocity is above a given threshold. The stability of these bunched states is based on the existence of two characteristic velocities in the coupled system. The result of analytical stability analysis is in excellent agreement with the results of numerical simulations.
\end{abstract}

Systems of coupled long Josephson junctions (LJJs) present intriguing possibilities as tunable fluxon oscillators, since various coupling mechanisms have proven to synchronize fluxon motion in the individual junctions. ${ }^{1-3}$ In particular, coupling mechanisms that are distributed along the spatial dimension of the junctions ${ }^{3-9}$ have proven not only to produce phase locking of fluxon motion, but also effects like mode dependent characteristic velocities ${ }^{9,10}$ and hyperradiance. ${ }^{11}$ These effects, explained by the usual sine-Gordon model for the LJJ, rise from the fact that the distributed coupling mechanism breaks the Lorentz invariance of the model and thereby introduces new phenomena to the dynamics. In this paper we will consider the inductive coupling between two LJJs. ${ }^{3-5,9}$ The stability of bunched states of fluxons is analyzed and it is found that the repulsive state of bunched unipolar fluxons may be stable when moving at high speed. This result, confirmed by numerical simulations, is closely connected to a splitting of the characteristic velocity of the coupled system. We show how this bunched state of unipolar fluxons will manifest itself in real experiments and we discuss the properties of the emitted power from this mode. The attractive state of a fluxon and an antifluxon is found to be stable regardless of speed, as is well known. ${ }^{9,11}$

The equations of motion for the field variables in the two LJJs are a $^{4,9}$

$$
\begin{gathered}
\phi_{x x}-\phi_{t t}-\sin \phi=\alpha \phi_{t}-\eta_{1}-\Delta \psi_{x x}, \\
\psi_{x x}-\psi_{t t}-\sin \psi=\alpha \psi_{t}-\eta_{2}-\Delta \phi_{x x}
\end{gathered}
$$

where the phases $\phi$ and $\psi$ represent the quantum mechanical phase difference over each of the junctions. The spatial dimension is normalized to the characteristic Josephson length and the time dimension is normalized to the inverse plasma frequency of the junctions. Tunneling of quasiparticles is described by the dissipative terms $\sim \alpha$ and the external bias current, normalized to the critical current of the junctions, is given by $\eta_{i}$. The magnetic coupling parameter, $\Delta$, is a coupling constant given by the geometry of the system $(0 \leq \Delta<1) .{ }^{4,9}$ The equations (2) describe a system of identical junctions. In reality, this cannot be obtained and some differences will be found in the characteristic lengths, times, and dissipation parameters of the two junctions. However, the purpose of the present paper is to demonstrate stability of certain modes in the coupled system and it is therefore a convenient choice to consider identical parameters for the two junctions. For a study of systems of different characteristic parameters see Ref. 5 .

The fundamental bunched soliton solution to the unperturbed system (i.e., for $\alpha=\eta_{i}=0$ ) is given by ${ }^{9}$

$$
\phi^{(b)}=4 \tan ^{-1}\left[-\exp \left\{\gamma\left(\frac{u}{\sqrt{1+\sigma \Delta}}\right) \frac{x-u t}{\sqrt{1+\sigma \Delta}}\right\}\right]
$$

where $\phi^{(b)}=\sigma \psi^{(b)}, u$ is the velocity of the wave, and $\gamma^{-1}(u)=\sqrt{1-u^{2}}$. This traveling wave solution to Eq. (2) can be sustained in the system for $\alpha \neq 0$ when $\eta_{1}=\sigma \eta_{2}$, where $\sigma= \pm 1$ denotes if the solution consists of fluxons of equal $(+1)$ or opposite $(-1)$ polarity. The above solution to the unperturbed system describes, however, only the special case where the two solitons have the same spatial coordinate. In general the solitons can be represented with individual parameters and, in the case when they are well separated in space, each soliton can be expressed (to first order in $\Delta$ ) by

$$
\phi^{(0)}=\sigma \psi^{(0)}=4 \tan ^{-1}[-\exp \{\gamma(u)(x-u t)\}] .
$$

By writing the energy of the system as ${ }^{9}$

$$
\begin{aligned}
H= & \int\left[\frac{1}{2} \phi_{x}^{2}+\frac{1}{2} \phi_{t}^{2}+1-\cos \phi\right] d x \\
& +\int\left[\frac{1}{2} \psi_{x}^{2}+\frac{1}{2} \psi_{t}^{2}+1-\cos \psi\right] d x+\Delta \int \phi_{x} \psi_{x} d x
\end{aligned}
$$

we can compare the energies of the different modes in the system by inserting the different solutions into the energy expression. We hereby obtain the energy of the bunched mode equation (2),

$$
H_{b}=16 \sqrt{1+\sigma \Delta} \gamma\left(\frac{u}{\sqrt{1+\sigma \Delta}}\right) .
$$

The energy of the mode of separated solitons is given (to first order in $\Delta$ ) by $^{5}$ 


$$
H_{s}=16 \gamma(u)+8 \sigma \Delta \gamma(u) \frac{r \gamma(u)}{\sinh r \gamma(u)}
$$

where $r$ is the spatial separation between the kinks. From this expression, we clearly see that kinks of equal polarity $(\sigma=1)$ will repel each other while kinks of opposite polarity will attract each other. This interaction between fluxons has been verified in Refs. 3, 9, and 11 for fluxons of opposite polarity. However, the simple energy expression Eq. (6) does not capture the complicated wave nature of the problem. It appears, as we will see below, that the bunched state of equal polarity fluxons $(\sigma=1)$ is in fact stable in the high velocity regime.

The origin of the stability of the bunched $\sigma=1$ mode can be found in the multiple characteristic velocity nature of the coupled equations (1). Writing the equations for the sum, $\mu=\phi+\psi$, and the difference, $\nu=\phi-\psi$, of the two fields, we obtain,

$$
\begin{aligned}
& (1+\Delta) \mu_{x x}-\mu_{t t}-2 \sin \frac{\mu}{2} \cos \frac{\nu}{2}=\alpha \mu_{t}-\eta_{1}-\eta_{2}, \\
& (1-\Delta) \nu_{x x}-\nu_{t t}-2 \sin \frac{\nu}{2} \cos \frac{\mu}{2}=\alpha \nu_{t}-\eta_{1}+\eta_{2} .
\end{aligned}
$$

As can be seen directly from Eqs. (7) and (8) the $\mu$ and the $\nu$ fields evolve on different time scales, $\nu$ being limited by $u_{*}=\sqrt{1-\Delta}$ and $\mu$ limited by $u_{\infty}=\sqrt{1+\Delta}$. For $\sigma=1\left(\eta=\eta_{1}=\eta_{2}\right)$ we can now analyze the stability of the traveling bunched mode equation (2), by setting $\mu=2 \phi^{(b)}$ and consider $|\nu| \ll 1$ in order to linearize Eq. (8). In the following, we will assume $\sigma=1$ unless otherwise specified. For a traveling steady state of two bunched solitons we then obtain

$$
\mu_{\xi \xi}-2 \sin \frac{\mu}{2}=-\frac{u \alpha}{\sqrt{1-u^{2}+\Delta}} \mu_{\xi}-2 \eta
$$

where

$$
\xi=(x-u t) / \sqrt{1-u^{2}+\Delta} .
$$

Using the adiabatic perturbation technique, ${ }^{12}$ Eqs. (7) and (9) give the power balance velocity of the bunched mode,

$$
u=\sqrt{1+\sigma \Delta} \frac{\pi \eta}{4 \alpha} / \sqrt{1+(\pi \eta / 4 \alpha)^{2}} .
$$

Having fixed the overall motion of the bunched soliton pair by the power balance, we set $\alpha=0, \eta_{1}=\eta_{2}$ in Eq. (8) and describe the internal motion of the bunched solitons by an angular frequency $\omega$, given by

$$
\partial / \partial t=(\partial \xi / \partial t) \partial / \partial \xi+i \omega .
$$

Using the identity $\cos \phi^{(b)}=1-2 \operatorname{sech}^{2} \xi$, we obtain the following equation from Eq. (8):

$$
\lambda \zeta_{\xi \xi}-\left\{\kappa-2 \operatorname{sech}^{2} \xi\right\} \zeta=0
$$

where

$$
\begin{aligned}
& \lambda=\frac{1-u^{2}-\Delta}{1-u^{2}+\Delta} \\
& \kappa=1-\frac{1-\Delta}{1-u^{2}-\Delta} \omega^{2} \\
& \nu=\zeta(\xi) \exp (-\varepsilon \xi) \\
& \varepsilon=i u \omega \frac{\sqrt{1-u^{2}+\Delta}}{1-u^{2}-\Delta}
\end{aligned}
$$

First we note that the exponential factor in Eq. (16) is a phase factor which does not affect the stability analysis. Equation (13) represents the eigenvalue problem for the stability of the bunched pair. For $\lambda>0$ we observe a potential well in the equivalent Schrödinger problem, where $\omega^{2}<0$ implies instability. $\lambda$ changes sign when $u^{2}$ exceeds $1-\Delta$, and thus changes the potential well into a barrier. The solutions are all extended waves and constitute Cherenkov radiation. In this case all solutions to Eq. (13) do not diverge and the bunched state is stable. Detailed analysis follows in a longer publication elsewhere.

We conclude that the bunched state of two unipolar fluxons is stable in magnetically coupled long Josephson junctions if the normalized velocity of the mode is in the interval,

$$
\sqrt{1+\Delta}=u_{\infty}>|u|>u_{*}=\sqrt{1-\Delta}
$$

and unstable otherwise. From Eqs. (11) and (19) the bias current density necessary for a stable bunched state is found to be

$$
1>|\eta|>\eta_{*}=2 \sqrt{2} \alpha \pi^{-1} \sqrt{\Delta^{-1}-1} .
$$

Since stable fluxon propagation in dc driven junctions is only possible for $|\eta|<1$ we can further obtain a minimal value of the magnetic coupling parameter for which the bunched state of unipolar fluxons can be stable. From Eq. (19) we find that the coupling parameter must obey the following condition in order to make the unipolar bunched state possible:

$$
\Delta>\Delta_{\mathrm{cr}}=8 \alpha^{2} /\left(8 \alpha^{2}+\pi^{2}\right) .
$$

The stability of the bunched mode of unipolar fluxons has been verified by numerical simulations of the system defined by Eq. (2) with periodic boundary conditions,

$$
\phi(0, t)=\phi(L, t)+2 \pi, \quad \psi(0, t)=\psi(L, t)+2 \pi \sigma
$$

where $L$ is the length of the system. In order to simulate an infinite system size we have chosen $L=20$ for all our simulations. The bias current density, $\eta=\eta_{1}=\eta_{2}$, is identical for the two junctions $(\sigma=1)$. In Fig. 1 we show a typical normalized current-voltage $(I-V)$ curve for the system, where the normalized voltage $V$ is given by the fluxon velocity, $u=V L / \pi$. The $I-V$ curve is here shown for $\Delta=0.2$ and $\alpha=0.1$. We initiated the system at high bias current $(\eta)$ with a soliton in each system separated by $d x=0.05$ in space. After a transient time of 1000 normalized time units, we measured the voltage $\left(V=\left\langle\phi_{t}\right\rangle\right)$ across the junctions. In changing the bias current by $\delta \eta=0.001$ we introduced a small disturbance in the $\phi$ field in order to break a perfect symmetry between the two systems. In this way we made sure that any instability of the current mode would present itself. As can be seen from Fig. 1 the high bias situations show a soliton speed with asymptote $u_{\infty}=\sqrt{1+\Delta}$, inherent for the bunched state equation $(2)(\sigma=1) \cdot{ }^{9,13}$ In decreasing the bias current we see that the bunched state continues to exist until the velocity $u_{*}=\sqrt{1-\Delta}$ is reached. At this point there is a jump in the $I-V$ curve implying splitting 


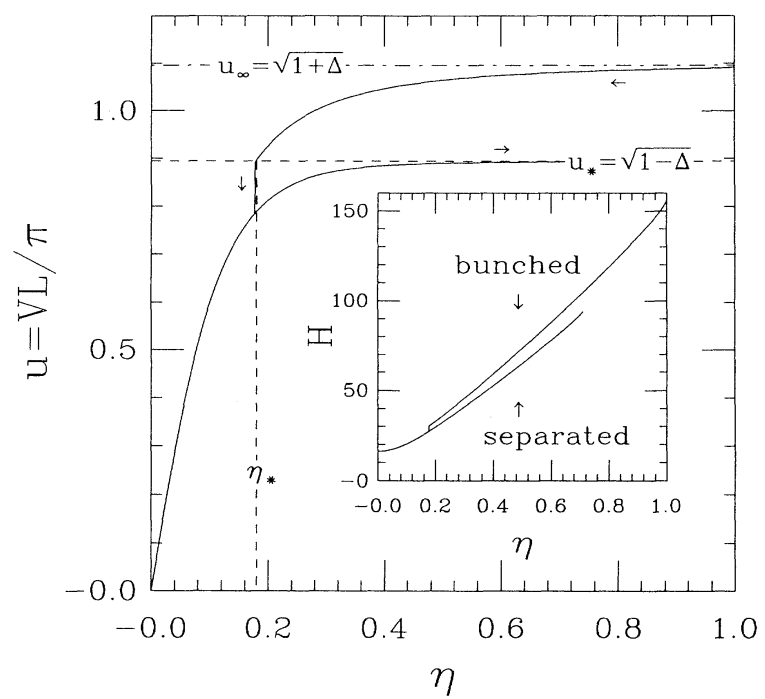

FIG. 1. The normalized fluxon velocity $u$ as a function of the normalized bias current density $\eta$. Parameters are $\alpha=0.1, \Delta=0.2, L=20$, and $\sigma=1$. The dash-dotted line indicates the asymptotic velocity $u_{\infty}$ for the bunched traveling wave solution equation (2) for $\sigma=1$. The dashed lines indicate the boundary for the stability of the bunched mode as given by Eqs. (18) and (19). The inset shows the total energy of the system as given by Eq. (4).

of the solitons from the bunched mode to the separated mode. The separated solitons have a shape roughly given by Eq. (3) and do therefore have a smaller velocity for the given bias current. Increasing the bias current from below $\eta_{*}$ the solitons stay separated as predicted by the system energy equation (6), that shows that the energy of the bunched state is always larger than the energy of the separated state when $\sigma=1$. This fact is confirmed by calculating the total energy equation (4) from the numerical solutions. We have shown the energy in the inset of Fig. 1, where the energy of the bunched state clearly is higher for all values of the bias current where the bunched state exists as a stable object. Even at the transition point, $\eta_{*}$, the energy of the bunched state is higher than that of the separated state. From Eqs. (5) and (6) this difference in energy can be estimated to be (first order in $\Delta$ )

$$
H_{b}-H_{s} \approx 8 \Delta \sqrt{1+(\pi \eta / 4 \alpha)^{2}} .
$$

We have tested the criterion for the stability of the bunched mode for many different parameter sets. In Fig. 2 we have shown the switching points in bias current for two different dissipation parameters and for varying coupling strength. The results of the numerical simulations are shown as markers and the analytical results Eqs. (19) and (20) are shown as solid curves. The markers ( $\square$ ) represent simulations with $\alpha=0.1$ and the markers $(\diamond)$ represent $\alpha=0.05$. As is evident from this figure, the analytical expression fits the numerical data excellently. The only deviation between the numerical data and the analytical expression is for low $\Delta$ and high $\alpha$. This deviation is quite understandable within the framework of the

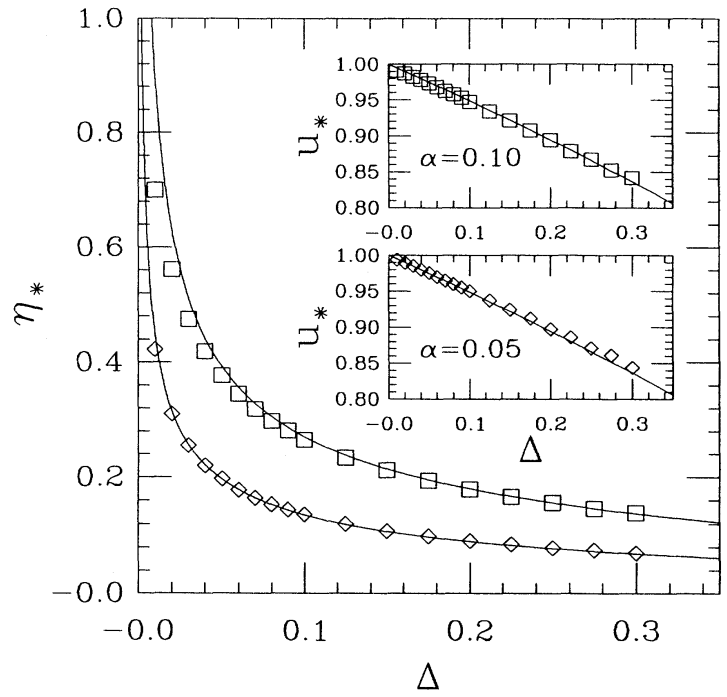

FIG. 2. The lower limit in bias current, $\eta_{*}$, as a function of the coupling strength $\Delta$. Parameters are $L=20$ and $\sigma=1$. The markers indicate the results of numerical simulations; $\square$ : $\alpha=0.1 ; \diamond: \alpha=0.05$. The solid lines represent the analytical expression Eq. (19). The insets show the comparison between the numerical data (markers) and the analytical expression for the critical velocity equation (18).

adiabatic perturbation technique ${ }^{12}$ since we have used the unperturbed solution equation (2) to calculate the power balance between $\eta$ and $\alpha$ in order to arrive at Eq. (20). For high bias values the real kink solution is not well described by Eq. (2) and consequently, the analytical result does not agree completely with the numerical data. However, looking at the insets of Fig. 2 we see that the velocity criteria for the existence of the bunched state is verified completely even for small values of $\Delta$ (high values of $\eta$ ).

In conclusion, we have found a criteria for the stability of bunched unipolar fluxon modes in magnetically coupled Josephson junctions. These states, unstable when moving at low velocities, have been found to be stable above the characteristic velocity, $u_{*}=\sqrt{1-\Delta}$, even though the energy of the bunched state has been shown to be larger than the energy of the state of separated fluxons. The origin of the stability of these states has been understood as a result of a mismatch between the translational velocity of the mode and the velocity at which a small deviation from the bunched state can propagate. It is important to realize that this mechanism for bunching is different from other binding mechanisms encountered in the sine-Gordon system. Bunching of attractive fluxons is based solely on the energy ${ }^{5,9}$, and bunching of fluxons belonging to the same system is due to details in the friction forces. ${ }^{14}$

The stability of bunched unipolar fluxons is also important for the study of propagation of flux flows in magnetically coupled long Josephson junctions. This has previously been studied analytically for the repulsive interaction between the fluxons, ${ }^{15}$ but not for the bunched mode as described in this paper. 
We note that the stability of the attractive mode $(\sigma=$ $-1)$ can be found to be stable for all relevant velocities $(0 \leq|u|<\sqrt{1-\Delta})$ by use of the above stability analysis. This is consistent with the energy consideration, since the attractive bunched state is always a lower energy state than that of the separated state [see Eqs. (5) and (6)]. However, a simple energy argument cannot be used to prove the stability of the $\sigma=1$ mode, since no good ansatz for the kink profiles is known when the fluxons are close, but not identical.

Let us finally comment on the possible output effect emitted from the bunched modes. In Ref. 11 it was demonstrated that the attractive bunched mode $(\sigma=$ -1) can exhibit hyperradiance, i.e., the bunched state can emit more than four times the power than a single fluxon state can at a given frequency (velocity). This result is based on the fact that the $\sigma=-1$ state has its asymptotic velocity decreased from that of the single soliton state. Since the $\sigma=1$ state shows a larger asymptotic velocity compared to that of the single soliton state, we then conclude that the bunched unipolar fluxon mode cannot exhibit hyperradiance-in fact, it may not even be able to show superradiance.

We wish to thank P. Barbara for useful discussions. This work was performed under the auspices of the U.S. Department of Energy.
${ }^{1}$ S. Pagano, R. Monaco, and G. Costabile, IEEE Trans. Mag. MAG-25, 1080 (1989); R. Monaco, N. Grønbech-Jensen, and R. D. Parmentier, Phys. Lett. A 151, 195 (1990).

${ }^{2}$ M. Cirillo, I. Modena, F. Santucci, P. Carelli, and R. Leoni, Phys. Lett. A 167, 175 (1992).

${ }^{3}$ T. Holst, J. B. Hansen, N. Grønbech-Jensen, and J. A. Blackburn, Phys. Rev. B 42, 127 (1990); IEEE Trans. Mag. 27, 2704 (1991).

${ }^{4}$ M. B. Mineev, G. S. Mkrtchyan, and V. V. Shmidt, J. Low Temp. Phys. 45, 497 (1981); A. F. Volkov, Pis'ma Zh. Eksp. Teor. Fiz. 45, 299 (1987) [JETP Lett. 45, 376 (1987)].

${ }^{5}$ Yuri S. Kivshar and Boris A. Malomed, Phys. Rev. B 37, 9325 (1988); Niels Grønbech-Jensen, Ole H. Olsen, and Mogens R. Samuelsen, Phys. Lett. A 179, 27 (1993).

${ }^{6}$ Yuri S. Kivshar and Boris A. Malomed, Rev. Mod. Phys. 61, 763 (1989).

${ }^{7}$ Niels Grønbech-Jensen, Peter S. Lomdahl, and Mogens R. Samuelsen, Phys. Rev. B 48, 6353 (1993); Stanford P. Yukon and Nathaniel Chu H. Lin, IEEE Appl. Supercond 3, 2532 (1993).
${ }^{8}$ Boris A. Malomed, J. B. Khalfin, and B. Ya. Shapiro, Phys. Rev. B 48, 7348 (1993); Solid State Commun. (to be published).

${ }^{9}$ N. Grønbech-Jensen, M. R. Samuelsen, P. S. Lomdahl, and J. A. Blackburn, Phys. Rev. B 42, 3976 (1990).

${ }^{10}$ K. L. Ngai, Phys. Rev. 182, 555 (1969); P. Barbara and G. Costabile (unpublished); A. V. Ustinov, H. Kolstedt, M. Cirillo, N. F. Pedersen, G. Hallmanns, and C. Heiden, Phys. Rev. B 48, 10614 (1993).

${ }^{11}$ Niels Grønbech-Jensen and James A. Blackburn, Phys. Rev. Lett. 70, 1251 (1993); J. Appl. Phys. 74, 6432 (1993).

${ }^{12}$ D. W. McLaughlin and A. C. Scott, Phys. Rev. A 18, 1652 (1978).

${ }^{13}$ S. Sakai, P. Bodin, and N. F. Pedersen, J. Appl. Phys. 73, 2411 (1993).

${ }^{14}$ P. S. Lomdahl, O. H. Soerensen, and P. L. Christiansen, Phys. Rev. B 25, 5737 (1982).

${ }^{15}$ Yuri S. Kivshar and Tatyana K. Soboleva, Physica B 165166, 1651 (1990). 